tached at $G$ is omitted from the drawing in order to show parts otherwise concealed.

The essential parts are the platform and shelf. The platform, $A$, which measures $9 \frac{1}{2}$ by 7 inches is supported on legs having leveling screws, $B$, and has a portion cut out, $C$, on the longer side similar in form to the open space between the sides of the horseshoe base of a microscope. This opening is to admit light from an ordinary microscope mirror suspended beneath the stand by a jointed arm, $J$, allowing lateral motion and which is in turn attached to a horizontal rod sliding back and forward in a tube on the under surface of the platform. On the front edge of the platform bridging the light opening is the shelf, $E$, supported by two pillars, $D$. The dissecting apparatus is clamped to this shelf by the screw, $F$. The microscope may be firmly secured to the platform by the clamps, $H$, and holes are drilled in the platform to accommódate various positions of the microscope, but frequently the use of the clamps is unnecessary.

Dr. Chambers has suggested that I call attention to a useful improvement of his own dissecting apparatus introduced by $\mathrm{E}$. A. Thompson, of Amherst, Mass. Fine springs placed around the screws which move the needle carriage as at $I$ in the figure prevent lost motion and thus steady the initial motion of the needle which is a marked advantage in the finer work.

\section{WESLEYAN UNIVERSITY}

H. B. GOODRICH

\section{GASTRIC RESPONSE TO FOODS 1}

IX. THE INFLUENCE OF WORRY ON GASTRIC DIGESTION

ThE study of the influence of emotional strain on digestion in man offers some diffculties due to the fact that the emotions can not be readily controlled, nor are the subjects of extreme emotion readily amenable to experimentation. We were, however, able to obtain an interesting illustration of the pro-

1 The expenses of this investigation were defrayed by funds furnished by Mrs. M. H. Hendermon. found effect of mental anxiety on gastric digestion in the case of a first-year medical student who had previously served as a subject of gastric tests and whose stomach had been found entirely normal. This man was given one hundred grams of fried chicken on the morning of an important examination in chemistry, and was asked to write out his answers during the course of the test. $\mathrm{He}_{\theta}$ was plainly worried over the outcome of the examination and of his year's work. The resultant effect upon gastric digestion in prolonging evacuation for over two hours, with high intra-gastric acidity is charted in the figure. The same chart gives the normal

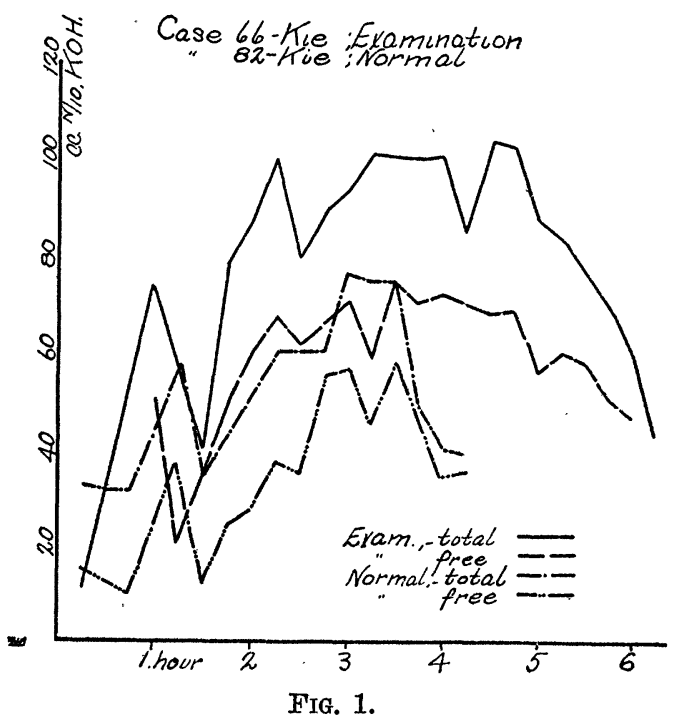

digestion curve for fried chicken on this subject as obtained a week later under the best mental conditions.

The experiments were carried.out by withdrawing samples from the stomach of the subject with the Rehfuss stomach tube at flfteen-minute intervals until the stomach was compty. The specimens were analyzed for total acidity and free hydrochloric acid and results expressed as c.c. of $\mathrm{N} / 10$ alkali roquired to neutralize 100 c.c. of sample. ${ }^{2}$

2 Fishback, Smith, Bergeim, Lichtenthaeler, Rehfuss and Hawk, Am. Jour. Physiol., 1919, XLIX., 174, and later communications. 
Pavlov, Cannon, Bickel and Sasaki ${ }^{3}$ and others have shown that in animals fear and rage may exert a most pronounced inhibiting effect on gastric peristalsis and secretion. It is also known that the clinical symptoms of gastric ulcer may be aggravated by emotional excitement due apparently to delayed evacuation associated with the hypersecretion of gastric juice commonly found in such cases. Our experiment is a clear-cut demonstration of a purely emotional dyspepsia and may serve as an additional emphatic warning to us all not unnecessarily to carry our troubles with us to the dinner table.

\section{RAYMond J. Miller, Olaf Bergeim, Philip B. Hawk}

Jumperson Medical College

\section{THE AMERICAN CHEMICAL SOCIETY. VI}

Problems in specifications for reagent chemicals: W. D. CoLlins. The following suggestions are offered as a basis for discussion: (1) The American Chemical Society should establish and publish specifications for chemical reagents. (2) Strength and purity should be prescribed as high as is consistent with good manufacturing practise. (3) The method of determining strength should be fully described. (4) Only impurities likely to be present should be considered. (5) For nearly all impurities a test should be described which will give no result with a satisfactory chemical. (6) The specifications should contain no provisions for penalties or premiums for variations from the strength and purity prescribed. (7) The specifications should not in general demand the purity required for work of the very highest refinement. (8) Specifications for containers are not necessary. (9) Names of manufacturers or brands should not be used in the specifications.

The standardization of laboratory apparatus and instruments in respect to quality, shape, size and packing: Thomas B. Freas. The manufacture of

8 Pavlov, "The Work of the Digestive Glands," London, 1902; Bickel and Sasaki, Deutsch. med. Wochensohr., 1905, XXXI., 1289; Cannon, "Bodily Changes in Pain, Hunger, Fear and Rage," New York, 1920. apparatus in this country is necessary in order to produce enough qualified skilled workers and experts to aid in times of national emergency. The cost of labor being high, the quantity basis of manufacture is urged. The splitting of endeavor is caused by the manufacture of too small quantities of any particular piece of apparatus. Quality, shapes and sizes of glassware, porcelain ware, rubber goods, woodenware, and platinum need standardizing. Possibly the number of shapes and sizes, at least, could be reduced to such an extent that the output per individual article would be materially increased. Reasons for packing in standard packages are also given and it is shown that this will tend to reduce overhead, especially in the dealer's business and allow a consequent reduction to the consumer. It is proposed to have a standardization office with a draftsman, the expenses of this office to be met by a small fee upon all the apparatus bearing the stamp of the committee of this society. If this small fee does not seem feasible, then some scheme should be adopted by which the Bureau of Standards would be able to carry out the ideas of standardization.

Recovery of the grease from the soapy wash waters in laundering: I. N. KUGELMAss. On the average a twenty-five gallon first suds waste yielded about one half liter of fatty acids extracted by gravitational separation through naphtha.

A rapid soap dissolving-distributing system: I. NeWTON KUGELMASS. The soap-dissolving tank contains a forty-five degree inclined perforated support suspended near the top of the tank, automatically fed with soap flakes and the whole immersed in water. An electric stirring device hastens solution. The clear soap solution is distributed to washers by pipe lines. At each washer a gauge gives the volume of soap solution passing through it into the washing machine. Advantages: rapid solution, economy in soap, time, and labor, correct soap concentration in washers.

The recovery of iodine from kelp: MERLE RANDALL. This paper is a summary of a study of the leach liquors at the U. S. Kelp-Potash Plant at Summerland, Calif. Green kelp, such as is harvested on the Pacific coast, contains about 0.0016 per cent. of iodine. The kelp is dried in direct heat driers, and chared either in incinerators or in retorts. The char is leached with hot water, and potassium chloride and sodium chloride removed from the leach liquor in crystallizing evaporators. Iodine should remain in the mother liquors. The 